\title{
HISTOPATHOLOGICAL CHANGES IN MICE INFECTED WITH RIVER WATER CONTAMINATED BY PASTEURELLA MULTOCIDA TYPE B:2
}

\author{
${ }^{1,5}$ Mohammed Muqdad Khaleel, ${ }^{1,3}$ Faez Firdaus Jesse Abdullah, ${ }^{1,6}$ Lawan Adamu, \\ ${ }^{2,7}$ Yusuf Abba, ${ }^{1,3}$ Abdul Wahid Haron, ${ }^{2}$ Mohd Zamri Saad and ${ }^{4}$ Abdul Rahman Omar \\ ${ }^{1}$ Department of Veterinary Clinical Studies \\ ${ }^{2}$ Department of Veterinary Pathology and Microbiology \\ Faculty of Veterinary Medicine, Universiti Putra Malaysia, 43400 UPM Serdang, Selangor, Malaysia \\ ${ }^{3}$ Centre for Ruminant Disease, Universiti Putra Malaysia, 43400 UPM Serdang, Selangor, Malaysia \\ ${ }^{4}$ Institute of Bioscience, Universiti putra Malaysia, Malaysia \\ ${ }^{5}$ Department of Microbiology, College of Veterinary Medicine, University of Mosul, Iraq, Mosul \\ ${ }^{6}$ Department of Veterinary Medicine, Faculty of Veterinary Medicine, \\ University of Maiduguri, PMB1069, Borno State, Nigeria \\ ${ }^{7}$ Department of Veterinary Pathology, Faculty of Veterinary Medicine, \\ University of Maiduguri, PMB1069, Borno State, Nigeria
}

Received 2014-03-03; Received 2014-03-05; Accepted 2014-03-28

\begin{abstract}
Haemorrhagic Septicaemia (HS) is a fatal systemic disease of bovines typified by an acute, highly fatal septicaemic disease with high morbidity and mortality. In this study, sixty five adult ICR mice were used for the study. In the first phase, 5 mice each were inoculated with $10^{9}$ cfu of $P$. multocida type B:2 through the intraperitoneal route. After development of classical signs of HS, the mice were euthanised and dropped into a tank of river waterfor 24, 48 and $72 \mathrm{~h}$. Contaminated water from the tank were collected at the three different times (24, 48 and $72 \mathrm{~h}$ ) and was used for intraperitoneal, oral and aerosol inoculation of 15 mice each with a dose of $1 \mathrm{~mL}^{-1} 10^{9} \mathrm{cfu}$ (intraperitoneal and aerosol) and $0.4 \mathrm{~mL}^{-1} 10^{9} \mathrm{cfu}$ (oral). The control group $(n=5)$ was given phosphate buffered saline. All mice in the three groups and control were culled after $72 \mathrm{~h}$ post inoculation and the vital organs (liver, spleen, lung, kidney, heart, brain) were collected in $10 \%$ buffered formalin, procesed, sectioned and stained with H\&E. Degeneration and necrosis, hemorrhage and infiltration of inflammatory cells were the most observed lesions in all the organs, while thrombosis and oedema were least observed. The lesion severity was moderate to severe using contaminated water at $72 \mathrm{~h}$ post contamination and mild to moderate when inoculated with water 24 and $48 \mathrm{~h}$ post contamination. Mice inoculated through the oral and intraperitoneal routes recorded more severe lesion compared with those inoculated through the aerosol route. However, using contaminated water at $24 \mathrm{~h}$, mice inoculated through the aerosol route had the highest distribution of inflammatory cells in the lungs, which declined following inoculation with contaminated water at 48 and $72 \mathrm{~h}$, respectively. This study has shown that contamination of water bodiesby carcasses of HS infected animals is a likely source of infection to heathy animals in the wild and that development of lesions severity is time dependant and significantly higher using contaminated water at $72 \mathrm{~h}$ than water contaminated for 24 and $48 \mathrm{~h}$. This further indicates the danger of leaving decomposing carcasses in water bodies for prolonged periods.
\end{abstract}

Keywords: Pasteurella Multocida type B:2; Mice, Contaminated River Water, Histopathology

Corresponding Author: Faez Firdaus Jesse Abdullah, Department of Veterinary Clinical Studies, Universiti Putra Malaysia, 43400 UPM Serdang, Selangor, Malaysia 


\section{INTRODUCTION}

Haemorrhagic Septicaemia (HS) is a fatal systemic disease of cattle and buffaloes typified by an acute, highly fatal septicaemic course with high morbidity and mortality. The disease is caused by serotypes B and E strains of Pasteurella multocida (Kharb and Charan, 2012; Khaleel et al., 2013; Abdullah et al., 2013a; 2013b). HS is widespread in most parts of tropical Asia and Africa. Even though the organism does not survive outside the animal body for a protracted period, it can survive up to several days in moist soil and water leading to extensive transmission during cloudburst season causing colossal financial losses (Jesse et al., 2013a). For infection to occur, the bacterial pathogen must have the capability of utilizing sufficient nutrients for optimal growth and multiplication whilst evading the host immune system. If the infection is to result in disease and cause cellular changes, the pathogen might also interact with the host in a way which might result in disturbance of homeostasis (Kharb and Charan, 2012). Pathogenesis of HS is not obviously tacit, nevertheless, it is indicated that during stress conditions bacteria proliferate and septicaemia develops leading to severe pathological alterations and death (Kharb and Charan, 2012; OIE, 2012). The primary virulence factors identified comprises the capsule, lipopolysaccharides, surface adhesions, iron acquisition proteins and ironregulated (Kharb and Charan, 2012). Furthermore, the proliferation of $P$. multocida and its associated pathology in mice via different routes of inoculation with HS contaminated river water is essential in the study of pathogenesis and transmission of HS. Therefore, the present work was set to evaluate the histopathological changes of $P$. multocida $\mathrm{B}: 2$ in different vital organs of mice following inoculation with $P$. multocida contaminated water through three different routes of inoculation.

\section{MATERIALS AND METHODS}

\subsection{Experimental Animals}

Sixty five healthy male adult mice of were used in this study. The mice were obtained from the Institute of Cancer Research (ICR) and kept at the Animal Resource Centre, Universiti Putra Malaysia. The study was approved by the institutional animal care and use commitee. The animals were confirmed negative for $P$. multocida following culture of peripheral blood for bacterial isolation. They were housed in plastic cages and provided with water and pellet ad libitum. Five mice were kept in each plastic cage for the control and treatment groups. The mice were observed for 2 weeks prior to the experiment to make sure that they acclimatize to the environment and were healthy.

\subsection{Bacterial Inoculation}

P.multocida B:2 used in this study were obtained from stock culture isolated from a previous outbreak of HS in the state of Kelantan, Malaysia. Identification of $P$. multocida was made using Gram-staining and biochemical test; oxidase, urea broth, Sulphur Indole Motility (SIM), Triple Sugar Iron (TSI) and citrate tests. The isolate was confirmed to be P.multocida type B:2 by the Veterinary Research Institute (VRI) Ipoh, Perak. Pure stock culture that was stored on nutrient agar slants was sub-cultured onto 5\% horse blood agar and incubated at $37^{\circ} \mathrm{C}$ for $18 \mathrm{~h}$. A single colony of P. multocida was selected and grown in Brain Heart Infusion Broth (BHI), incubated in shaker incubator at $37^{\circ} \mathrm{C}$ for $24 \mathrm{~h}$ before the concentration was determined by McFarland Nephelometer Barium Sulfate Standards. River water wasobtained from Hulu Langatriver and cultured to confirm that it was free from P. multocida type B:2. Fifteen mice were initially inoculated with $1.0 \mathrm{~mL}^{-1}$ of $10^{9}$ colony forming unit (cfu) of $P$. multocida type B:2 intraperitoneally. After 7-8 h of post inoculation, mice that survived were euthanized by cervical dislocation and the carcasses were placed in a tank containing river water. Five infected mice were placed in each tank for 24, 48 and $72 \mathrm{~h}$. One $\mathrm{ml}$ of the infected river water was inoculated intraperitoneally and via the aerosol routes while, 0.4 $\mathrm{mL}^{-1}$ was inoculated orally into five mice in each group respectively. The control group was inoculated with 1.0 $\mathrm{mL}^{-1}$ of sterile Phosphate Buffered Saline (PBS).

\subsection{Histopathology}

After $72 \mathrm{~h}$ of post inoculation, the mice were euthanized by cervical dislocation and the visceral organs (heart, kidney, lungs, spleen, brain and liver) were collected in $10 \%$ buffered formalin, processed, sectioned and stained with H\&E. Histopathological lesions were observed using light microscopy.

\subsection{Lesions Scoring and Statistical Analysis}

Histopathological changes observed (Degeneration and necrosis, inflammatory changes, heamorrhage, thrombosis and oedema) were scored based on the following categorization; 0 (normal), 1: Mild (less than $1 / 3$ of field involved), 2: Moderate (between $1 / 3$ and $2 / 3$ of field involved) and 3: Severe (more than $2 / 3$ of the field involved). Six microscopic fields were examined for each lesion per slide and the mean \pm standard 
deviation was calculated for each organ based on the different lesions observed.

\section{RESULTS}

The main histopathological lesions observed in the various organs were degenerative changes and necrosis, inflammatory changes marked by leucocytic infiltration, heamorrhage, thrombosis and oedema (Fig. 1a-f).

Degeneration and necrosis, haemorrhage and inflammatory changes were the most common lesions observed in all the organs following inoculation with river water contaminated with mice carcasses for $24 \mathrm{~h}$.
These lesions were mild to moderate in severity following oral, intraperitoneal and aerosol routes of inoculation. Thrombosis and oedema were mild in all the organs following all routes of administration. The kidney, spleen, heart and brain recorded higher lesion distribution after inoculation with contaminated river water that had stayed for $24 \mathrm{~h}$ (Fig. 2).

Following inoculation with contaminated water after $48 \mathrm{~h}$, degeneration and necrosis and inflammatory changes were the most frequently observed lesions, manifesting with mild and moderate severities among the various organs.
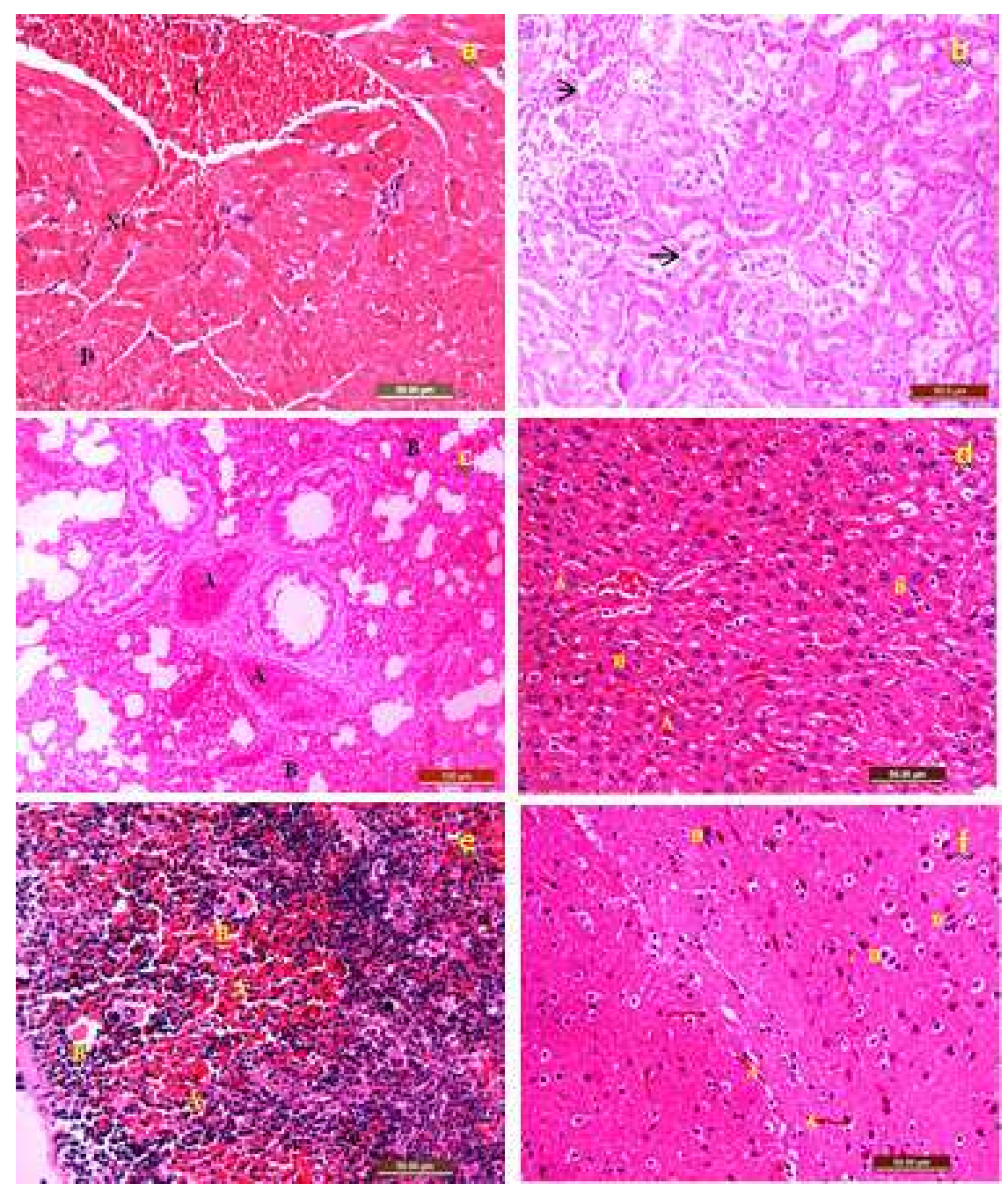

Fig. 1. Photomicrograph of the (a) heart showing congestion (C), necrosis (NC) and degeneration (D) (b) kidney showing heamorrhage and tubular necrosis (arrows) (c) lung showing areas of congestion (A) and oedema (B) (d) liver showing microthrombi (A) and hepatocytes degeneration (B) (e) spleen showing congestion of red pulp (A) and necrotic follicles in the white pulp (B) (f) brain showing vascular congestion (A) and neuronal necrosis (B), H\&E×400 
Heamorrhage and thrombosis were mild and less frequent in occurrence and severity, which was a similar observation during inoculation with contaminated water after $24 \mathrm{~h}$. However, oedema was observed more frequently among the various organs than it was following inoculation with contaminated water that had stayed for 24 h. Here, the kidneys and brain had the most lesion distribution when compared to other organs (Fig. 3).

Following inoculation with contaminated water that had stayed for $72 \mathrm{~h}$, degeneration and necrosis and inflammatory changes were the most commonly observed lesions in all the organs. The severity of these lesions were moderate to severe following oral and intraperitoneal routes, while aerosol route recorded lesions of mild to moderate severity. Oedema and heamorrhage were mild to moderate in severity, while thrombosis was mild and observed in the aerosol inoculated group only. Comparatively, the distributions of degeneration and necrosis, inflammatory cells and oedema were more in severity after using contaminated water that had stayed for $72 \mathrm{~h}$ than it was when inoculated with contaminated water that had stayed for 24 and $48 \mathrm{~h}$ (Fig. 4).

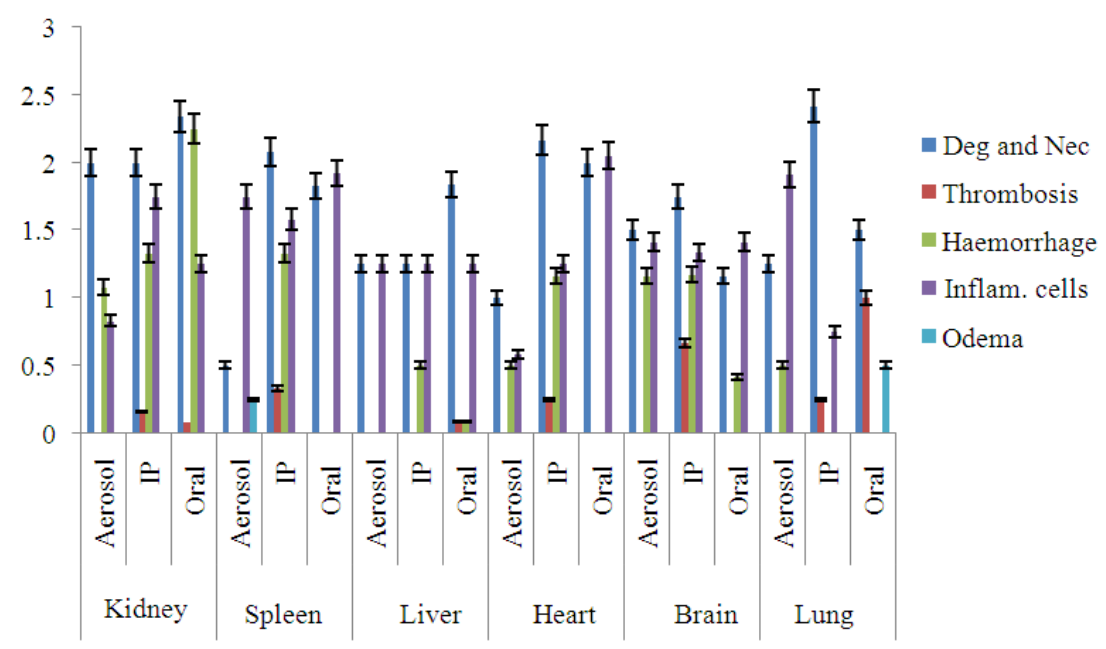

Fig. 2. Bar graph showing distribution of pathological lesions in organs of mice inoculated with water contaminated with infected mice carcass for $24 \mathrm{~h}$ via aerosol, intraperitoneal and oral routes. Inflam. Cells = inflammatory cells; Deg and Nec = Degeneration and Necrosis

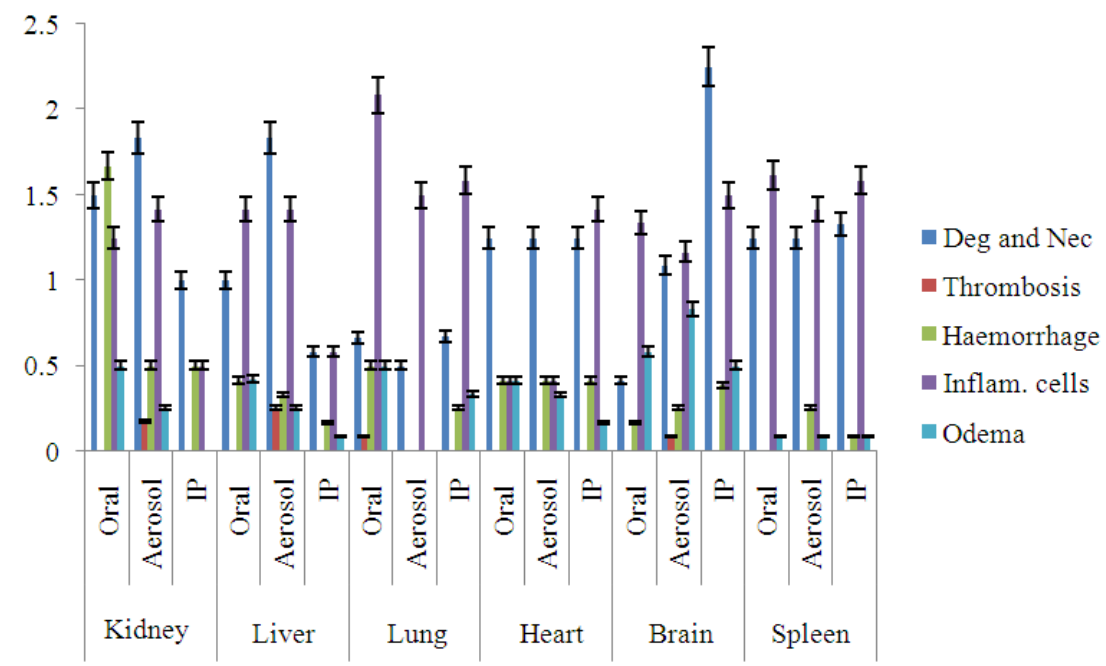

Fig. 3. Bar graph showing distribution of pathological lesions in organs of mice inoculated with water contaminated with infected mice carcass for $48 \mathrm{~h}$ via aerosol, intraperitoneal and oral routes. Inflam. Cells = inflammatory cells; Deg and Nec = Degeneration and Necrosis 


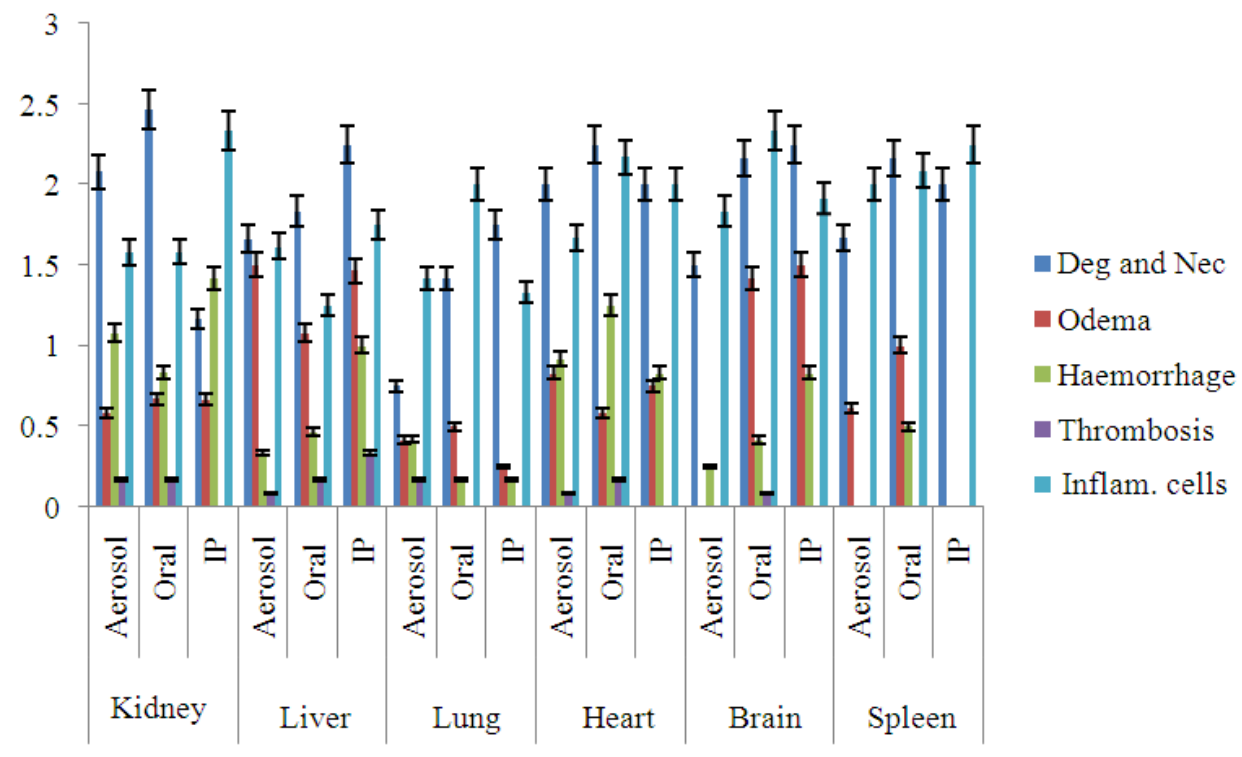

Fig. 4. Bar graph showing distribution of pathological lesions in organs of mice inoculated with water contaminated with infected mice carcass for $72 \mathrm{~h}$ via aerosol, intraperitoneal and oral routes. Inflam. Cells = inflammatory cells; Deg and Nec = Degeneration and Necrosis

\section{DISCUSSION}

In the past, several researchers have used rats and mice as a model animal for propagation of $P$. multocidatype B:2 (Affandi et al., 2012; Jamal et al., 2013; Faez et al., 2013). Although the routes of inoculation used in these studies differ in most cases, the clinical signs and pathological lesions observed were similar and classical to what was observed in natural HS infection. In this study, the possibility of infection transmission via river water was shown in order to mimic the natural scenario. It was observed that bacteria was shed into the water and was successfully recovered and inoculated into susceptible mice through various routes of inoculation. Earlier, Abdullah et al. (2013b) had reported the successful recovery of $P$. multocida using PCR from various organs of mice inoculated with contaminated river water. HS is a deadly infection and the extent of lesion manifestation depends on the duration of the disease and the dose of bacterial inoculums (Kharb and Charan, 2013). This is in line with the findings of this study, where moderate and severe lesions were observed following inoculation with contaminated water that had stayed for $72 \mathrm{~h}$ in comparison to lesions observed following inoculation with contaminated water that had stayed for 24 and $48 \mathrm{~h}$. This shows that the $P$. multocida was more active at $72 \mathrm{~h}$ post contamination, perhaps due to decomposition of the mice carcass and shedding of the bacteria into the water. The most common lesions observed were degeneration and necrosis, haemorrhage and inflammatory cells. However, the distributions of these lesions in various organs following three routes of inoculation differ. Similar observations were made by (Affandi et al., 2012; Faez et al., 2013) following oral inoculation of $P$. multocida in mice. Similarly, Jamal et al. (2013) reported histological evidence of hemorrhage in the lungs, kidney and liver of immune-suppressed rats exposed to $P$. multocida type B:2 in a related study, Jesse et al. (2013b) observed that calves inoculated intramuscularly and intravenously developed classical HS with presence of degeneration, necrosis, hemorrhage and inflammatory cells. It was observed that the severity of his to pathological lesions in this study was moderate to severe following intra-peritoneal and oral routes of inoculation. Kharb and Charan (2013) reported than regardless of the route of inoculation (intranasal or subcutaneous), mortality in mice was $80 \%$ in all the groups. In a related study in buffalo calves, Abubakar and Zamri-Saad (2011) observed that calves infected through the intra-tracheal route had more severe lesions which were related to the respiratory system, while those inoculated via the oral route manifested more severe enteric lesions. In our 
study, mice inoculated via the aerosol route had a moderate level of inflammatory cells in the lungs when compared with those inoculated via the oral and intraperitoneal routes, which had mild lesion severity. However, the lesion severity was mild following inoculation with contaminated water that had stayed for 48 and $72 \mathrm{~h}$, signifying lesser severity of the inflammatory process. The least commonly observed lesions at histopathology were thrombosis and oedema. These lesions were mild following inoculation with contaminated water that had stayed for $24 \mathrm{~h}$ and mild to moderate when inoculated with contaminated water that had stayed for 48 and 72 h. Jesse et al. (2013b) reported a similar finding in calves inoculated with $P$. multocida, where thrombosis was mild in all the groups.

\section{CONCLUSION}

This study has evaluated that contaminated river water does serve as a source of HS infection in the wild and that infection will manifest with characteristic histopathological lesions of HS. It also showed the importance of time and route of inoculation in the severity of lesions manifested following infection with water contaminated with infected mice carcasses. Limitations of this study were the short duration of the carcass in the water after death $(24,48,72 \mathrm{~h})$ and the frequency of inoculation of infected water to mice (once). Since in the wild, dead animal carcasses in water bodies stay for weeks or months and other animals constantly drink from the same source, we are unable to determine if a longer stay of the carcass in the water will result in more or less bacterial shedding and if increased frequency of inoculation of infected water will result in more extensive lesions at histopathology. Therefore, further studies should look at the possibility of having increased or decreased bacterial shedding from the infected carcass into the water with time and also if increased frequency of inoculation with the infected water will result in more extensive pathological lesions or not.

\section{ACKNOWLEDGEMENT}

We thank the staff of the Department of Veterinary Clinical Studies, Universiti Putra Malaysia and Research Centre for Ruminant Disease, in particular Yap Keng Chee, Mohd Jefri Norsidin and Mohd Fahmi Mashuri for their assistance. The project was funded by Ministry of Higher Education Malaysia.

\section{REFERENCES}

Abdullah, F.F.J., A.Y. Osman, L. Adamu, M.S.M. Yusof and A.R. Omar et al., 2013a. Polymerase chain reaction detection of Pasteurella multocida type B:2 fin mice following oral inoculation. Asian J. Anim. Vet. Adv., 8: 493-501. DOI: 10.3844/ajavsp.2013.146.151

Abdullah, F.F.J., M.M. Khaleel, L. Adamu, A.Y. Osman and A.W. Haron et al., 2013b. Polymerase chain reaction detection of Pasteurella multocida type B:2 in mice infected with contaminated river water. Am. J. Anim. Vet. Sci., 8: 146-151. DOI: 10.3844/ajavsp.2013.146.151

Abubakar, M.S. and M. Zamri-Saad, 2011. Clinicopathological changes in buffalo calves following oral exposure to B:2. Basic Applied Pathol., 4: 130135. DOI: 10.1111/j.1755-9294.2011.01113.x

Affandi, A.S., J.F.F. Abdullah, A.A. Saharee and J. Sabri, 2012. Clinical reponse and pathological vchanges associated with Pasteurella multocida typr B:2 infection through oral route inoculation in mice. Proceedings of the 7th Seminar of Veterinary Sciences, Feb. 2-Mar. 2, University Putra Malaysia.

Faez, F.J., S.A. Affandi, A.Y. Osman, L. Adamu, M. Zamri-Saad et al., 2013. Clinico-pathological features in mice following oral exposure to Pasteurella multocida type B:2. IOSR J. Agric. Veterinary Sci., 3: 35-39. DOI: 10.9790/2380 0343539

Jamal, H., M.H.M. Nazrul, N. Masyitah, A.A. Mahmood and I. Salmal, 2013. Alternative animal model for Pasteurella multocida and Haemorrhagic septicemia. Biomed. Res., 24: 263-266.

Jesse, F.F.A., L. Adamu, Y.O. Abdinasir, M.Z. Saad and Z. Zakaria et al., 2013a. Acute phase protein profiles and clinico-pathological changes in mice associated with the infection of Pasteurella multocida type B and the bacterial lipopolysaccharide and outer membrane protein immunogens. J. Anim. Veterinary Adv., 12: 186-193.

Jesse, F.F.A., L. Adamu, Y.O. Abdinasir, Z. Zakaria and R. Abdullah et al., 2013b. Clinicopathological responses of calves associated with infection of Pasteurella multocida type $\mathrm{B}$ and the bacterial lipopolysaccharide and outer membrane protein immunogens. Int. J. Anim. Veterinary Adv., 5: 190-198. 
Khaleel, M.M., F.F.J. Abdullah, L. Adamu, A.Y. Osman and A.W. Haron et al., 2013. Acute phase protein responses in mice infected with river water contaminated by Pasteurella multocida type $\mathrm{B}: 2$. Am. J. Anim. Veterinary Sci., 8: 159-164. DOI: 10.3844/ajavsp.2013.159.164
Kharb, S. and S. Charan, 2013. Mouse model of haemorrhagic septicemia: Dissemination and multiplication of Pasteurellamultocida B:2 in vital organs after intranasal and subcutaneous challenge in mice. Veterinary Res. Comm., 37: 59-63. DOI: 10.1007/s11259-012-9547-5

OIE, 2012. Haemorrhagic septicemia. Terrestial Manual. 